\title{
UNUSUAL PRESENTATION OF COVID 19 - A CASE REPORT
}

\author{
TASMINA CHOWDHURY TULI ${ }^{1}$, SADIA HALIMA², ARFA RAHMAN AVA ${ }^{3}$, QUAZI TARIKUL ISLAM ${ }^{4}$
}

\begin{abstract}
:
An acute respiratory disease caused by a new strand of coronavirus (SARS-CoV-2), which was ûrst identiûed in Wuhan, Hubei Province, China, quickly developed into a global pandemic. Common presentation associated with corona-virus disease 2019 (COVID-19) include fever, cough, and less commonly diarrhea, sore throat, chest pain, nausea, and vomiting, loss or attenuation of smell and taste sensation and palpitations. Till now observed sequelae are ARDS, multi organ dysfunction, thrombotic events and coagulation disorder, myocarditis. With time clinicians are observing emergence of new and unusual presentation, complications and sequelae of the disease. Our case report is of a middle aged man with typical symptoms of COVID 19 who ultimately developed right sided tension pneumothorax. Along with $\mathrm{O}_{2}$ therapy, anticoagulant, anti inflammatory drugs and other supportive treatment he was managed with water seal drainage. Though till date COVID 19 is not very well known to us but development of tension Pneumothorax as a sequelae is atypical as far observed which made this case interesting.
\end{abstract}

Key Words: Unusual presentation of COVID 19, Pneumothorax

Received: 05 March 2020

Accepted: 12 June 2020

DOI: https://doi.org/10.3329/bjm.v31i2.48539

\section{Introduction:}

Coronavirus disease 2019 (COVID-19), caused by severe acute respiratory syndrome coronavirus 2 (SARS-CoV-2), is a rapidly spreading disease causing increased morbidity and mortality across the globe. The World Health Organization declared the outbreak a pandemic on 11 March. ${ }^{1,2}$ As of 3 August 2020, more than 18 million cases of COVID 19 have been reported in more than 213 countries and territories, resulting in more than 689,000 deaths; more than 10.6 million people have recovered ${ }^{3}$.There is limited available knowledge regarding the natural history of the SARSCoV-2 infection. Other factors that are also making this infection spread like a pandemic include global travelers, lack of proven treatment, asymptomatic carriers, potential reinfection, underprepared global health care systems, and lack of public awareness and efforts to prevent further spread. People with COVID19 have had a wide range of symptoms reported ranging from mild symptoms to severe illness.
Symptoms may appear 2-14 days after exposure to the virus ${ }^{4}$. People with these symptoms may have COVID-19: fever or chills, cough, shortness of breath or difficulty breathing, fatigue, muscle or body aches headache, new loss of taste or smell, sore throat , congestion or runny nose nausea or vomiting, diarrhea ${ }^{5}$. COVID-19 can provisionally be diagnosed on the basis of symptoms and confirmed by reverse transcription polymerase chain reaction (RT-PCR) testing of infected secretions or CT imaging of the chest. ${ }^{6,7}$ are under investigation for COVID-19. Treatment is supportive. None has yet been shown to be clearly effective on mortality in published randomised controlled trials. ${ }^{8}$ However, remdesivir may affect the time it takes to recover from the virus. ${ }^{9}$ Depending upon the severity, oxygen therapy, intravenous fluids, and breathing support may be required.10 The safety and effectiveness of convalescent plasma as a treatment option requires further research. ${ }^{11}$.Other trials are investigating

1. Registrar of Medicine, Popular Medical College Hospital, Dhaka

2. Asstt. Registrar of Medicine, Popular Medical College Hospital, Dhaka

3. Asstt. Registrar of Medicine, Popular Medical College Hospital, Dhaka

4. Professor of Medicine, Popular Medical College Hospital, Dhaka- 1205, Bangladesh

Address of Correspondence: Dr.Tasmina Chowdhury Tuli, Registrar of Medicine, Popular Medical College Hospital, Dhaka

Bangladesh J Medicine 2020; 31 : 102-106 
whether existing medications can be used effectively against COVID-19 or the immune reaction to it. On 16 June, the RECOVERY Trial group released a statement that their preliminary results show low dose dexamethasone reduces mortality in patients receiving respiratory support, ${ }^{12}$ hospital admitted patients should receive VTE prophylaxis as per the standard of care for other hospitalized adults ${ }^{13}$.

\section{Case summary:}

Mr. Kanchan Banik,56- year- old male admitted to $\mathrm{PMCH}$ with 7 days fever and dry cough . His highest recorded temperature was $102^{\circ} \mathrm{F}$ and there was initially dry cough which progressed in both intensity and frequency over the first week of illness. During this time patient did not complain of any altered sense of smell, sore throat, headache, diarrhoea, vomiting or nasal congestion or has any contact history with any COVID patient. His only known co-morbidity is bronchial asthma which was controlled with salmetarol and fluticasone combinaton inhaler. He was a smoker for the last 20 years. On $4^{\text {th }}$ day of starting symptoms RT-PCR for COVID-19 from Nasopharyngeal swab was done and that came Negative.

The patient was desaturating at as evidenced on pulse oximeter with a $\mathrm{SPO}_{2} 89-91 \%$ at room air patient got admitted to $\mathrm{PMCH}$ on 05.07.2020. His CBC was normal but CRP and s.ferritin and D-dimer were raised. CxR showed bilateral lung infiltrate at periphery and HRCT chest showed typical bilateral pneumonia suggestive of COVD 19 involving 65\% of lungs.Patient was leveled as a case of COVID 19 and was managed initially with oxygen 3L/M , Inj. Enoxaparin 60IU S/C 12 hourly, Oral Moxifloxacin $400 \mathrm{mg}$ once daily and other

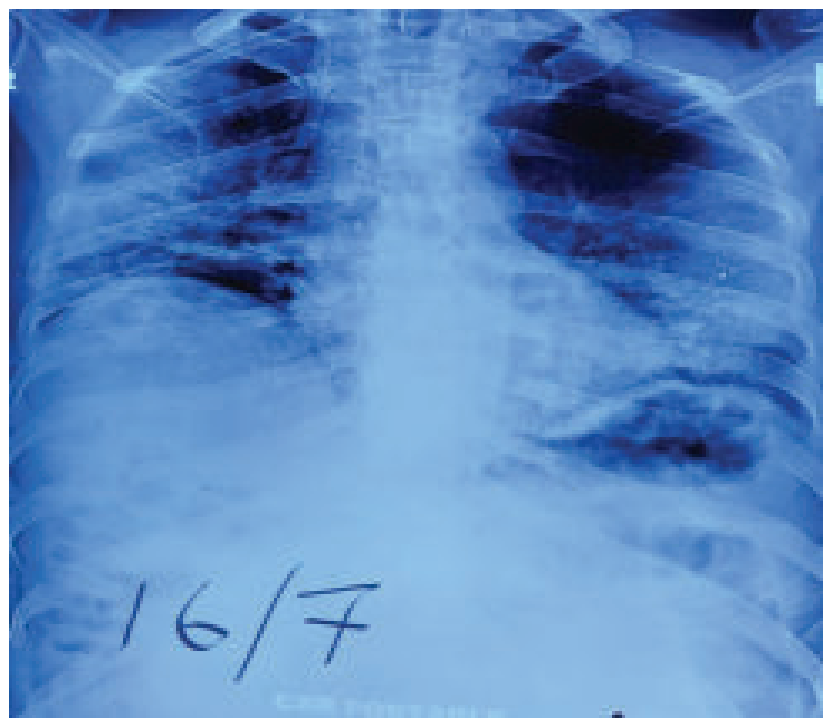

Fig 1a: Initial CXR shows bilateral peripheral pulmonary infiltrates supportive management. He became afebrile from this stage. However, patient developed intractable cough with productive sputum after admission.

Second RT PCR for COVID-19 on $12^{\text {th }}$ day was again negative. Patient started developing restlessness and progressively increased breathlessness, a simple face mask failed to maintain $\mathrm{O}_{2}$ saturation urged introduction of a Non-Rebreather Mask with 15LPM of oxygen. His inflammatory blood parameter was raising, CRP from 69 to $127 \mathrm{mg} / \mathrm{L}$, s ferritin from 512 to $1069 \mathrm{ng} / \mathrm{L}$ but CBC, D-dimer, S.procalcitonin, S.creatinine were normal and CxR showed increased bilateral shadow.

He was treated with injection Tocilizumab 2 doses on 12 hours apart according to his body weight considering cytokine storm syndrome. Along with this, patient was on corticosteroid support the entire course of illness. Initially, Inj. Dexamethasone $10 \mathrm{mg} 12$ hourly. The patient was put on Inj. Meropenam $1 \mathrm{gm}$ IV 8 hourly.

But despite all measures patient progressively became dyspnoic so an urgent chest $\mathrm{x}$-ray done which showed right sided pneumothorax . Second HRCT film, showed extensive B/L lung involvement with more organized shadows with development of tension pneumothorax on right side. Water seal drainage was inserted immediately and the symptoms relieve as evidenced by oxygen demand only 5-6 litres/min with Spo2 95\%.

During writing the case report patient is admitted in hospital with improving clinical features, blood parameters, radiology, maintaining $>95 \%$ saturation with 2 liter $\mathrm{O} 2 / \mathrm{min}$.

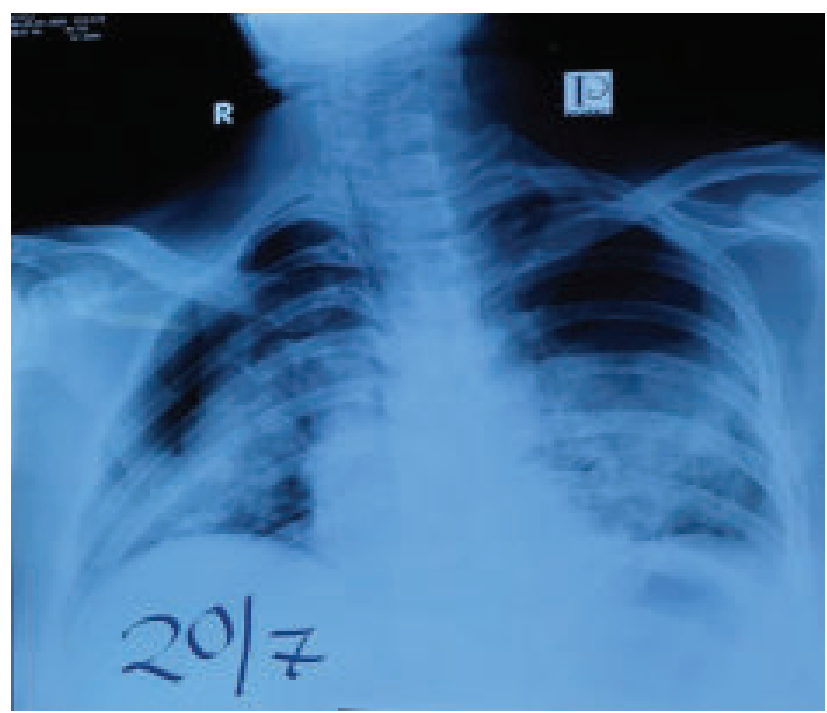

Fig 1b: CXR shows big consolidation of left and hydro pneumothorax on right 

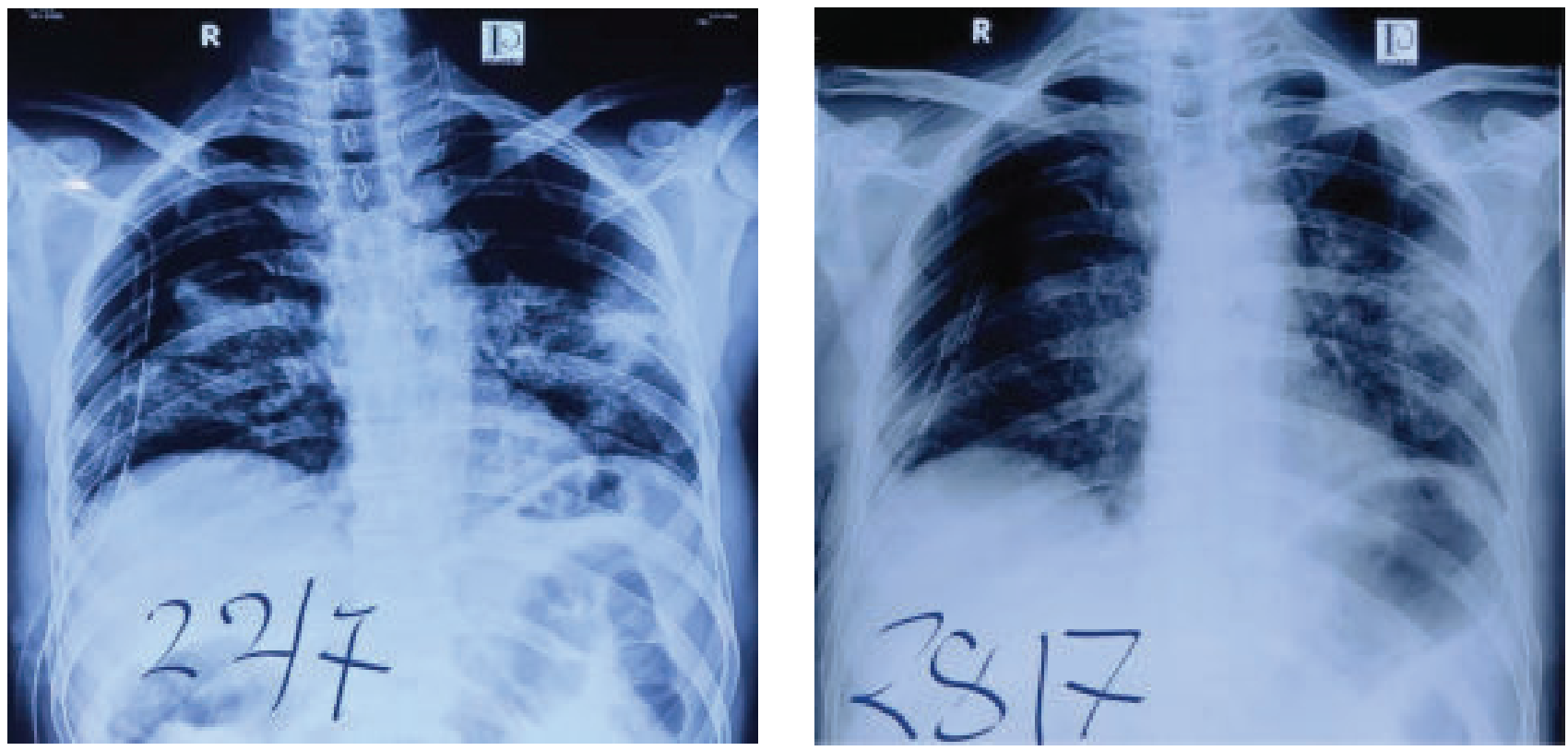

Fig 2(a\&b): Intercostal tube with improvement of radiological shadow
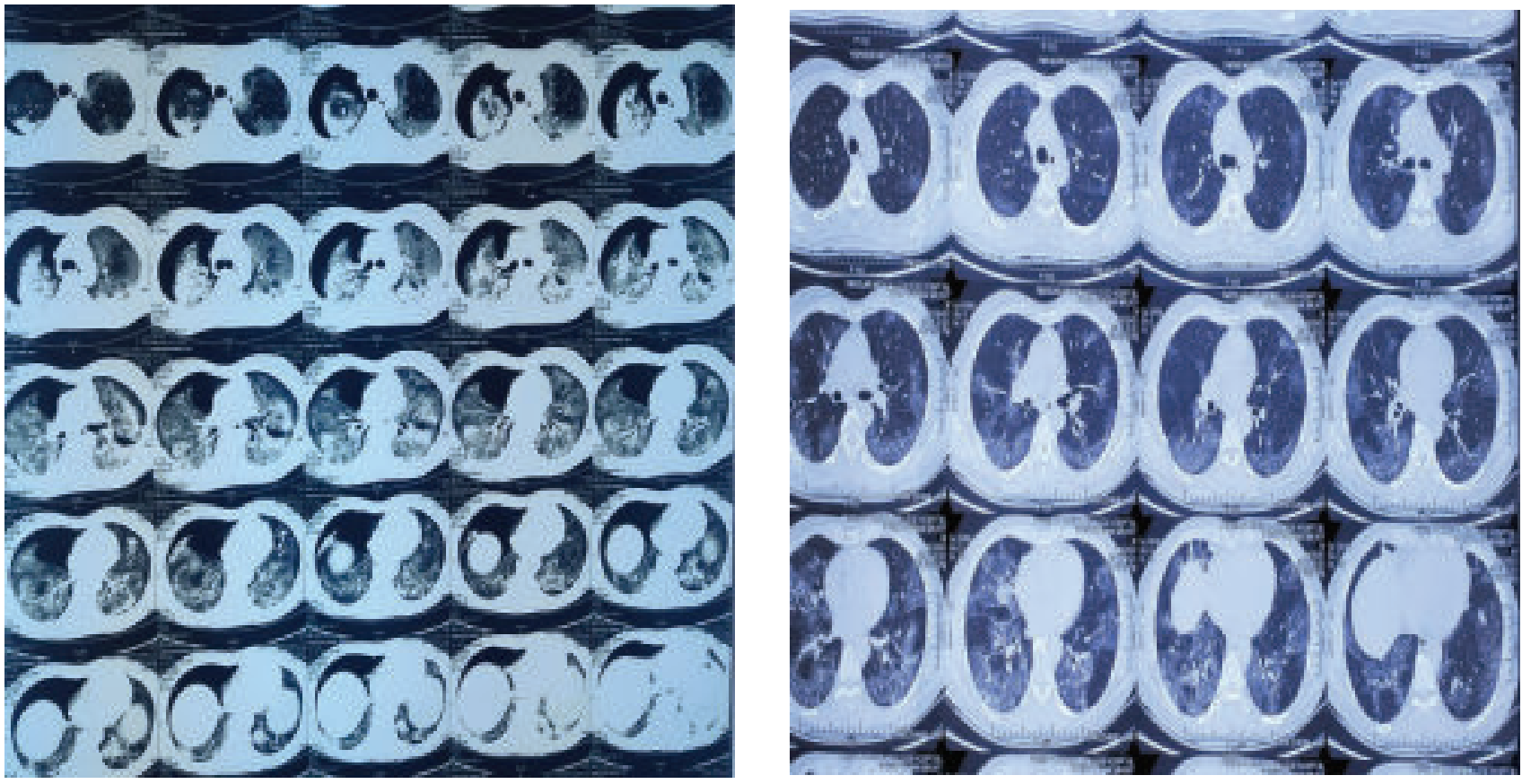

Fig 3 (a\&b): CT scn of chest Showing GGO and Pneumotorax 2 weeks later

\section{Discussion:}

coronavirus disease 2019 is a newly discovered viral disease whose knowledge is constantly evolving. Coronaviruses are described as enveloped, positive single-stranded RNA viruses, which have the ability to infect humans as well as a broad range of animals 13 . SARS-CoV-2 infects alveolar epithelial cells via receptor-mediated endocytosis using the angiotensinconverting enzyme II receptors in the lungs ${ }^{14}$. SARS-
CoV-2 has a wide variety of initial symptoms. Patients can range from asymptomatic infections to severe disease. Approximately 14\% (13.8\%, n =44,672) of patients develop severe disease, which requires hospitalization and oxygen support, and around 5\% require intensive care unit management 15 .

Despite being highly contagious, a significant portion of people infected with COVID-19 don't experience any symptoms. Those people who do exhibit symptoms 
most commonly experience dry cough, fever and difficulty breathing. However, there are also a myriad of atypical symptoms that may be under-reported ${ }^{16}$. The most commonly documented atypical symptoms include malaise, disorientation or exhaustion. A significant proportion of patients also experience the loss of smell and taste. About 10 percent of COVID-19 patients have gastrointestinal symptoms, such as diarrhea, vomiting and abdominal pain. Cardiovascular complications include dysrhythmias, venous thromboembolic events and myocardial infarction. COVID-19 also may cause clotting in blood vessels in the brain, increasing risk of ischemic stroke. A number of theories have been put forth to explain the unusual presentations of COVID-19. SARS-CoV-2 has been shown to directly infect many cell types and there's evidence that SARS-CoV-2 is neuro invasive. This would account for various neurological manifestations, including the loss of smell during the acute phase of the infection. An exaggerated immune response also may underlie atypical presentations in COVID-19 patients. Many patients who experience unusual symptoms exhibit a profound activation of their immune system. This response can produce a cytokine storm that can damage many organs, including the lungs, heart and brain. It's now clear that COVID-19 increases the risk of abnormal blood clotting. When clots form in small blood vessels, like in the extremities, minor skin symptoms can occur. But when clots occur in the lungs, heart or brain, more serious complications can develop, like pulmonary embolism, heart attack or stroke. They also may cause neurologic features, such as anosmia, encephalopathy and myopathy, In some cases, an overactive immune system has been shown to trigger autoimmune conditions in COVID-19 patients about two to three weeks after infection. Such neurological autoimmune conditions include: Bickerstaff encephalitis/GuillainBarré syndrome, acute disseminated encephalomyelitis and rhabdomyolysis ${ }^{16}$

Unusual presentations of COVID-19 pneumonia on CT scans with spontaneous pneumomediastinum and loculated pneumothorax a report of two cases and a review of the literature.) spontaneous pneumomediastinum (SPM) and Loculated pneumothorax (LPNX) are both generally rare clinical and radiological conditions associated with coronavirus Disease 2019 (COVID-19) ${ }^{16}$. There is report for the first time clinical data and radiological chest CT imaging of two patients affected by COVID-pneumonia associated with early radiological findings of SPM and LPNX. Most of the cases of SPM and PNX described in patients with COVID-19 pneumonia and in those affected by SARS have some features in common, including the absence of smoking history. Aggressive steroid therapy has also been speculated to play a role in the pathogenesis of spontaneous PNX in SARS patients due to the fact that steroids may delay wound healing and perpetuate air leakage. However, other studies have not confirmed this theory because steroids are useful in controlling the rapid and damaging host inflammatory response that is usually seen in viral pneumonia. More frequently, SPM associated with PNX in patients with COVID-19 have been complications of tracheal intubation or mechanical ventilation in patients with chronic obstructive pulmonary disease that needed invasive ventilation for correcting hypoxemia.

Our patient though RT PCR negative showed typical features of COVID 19 during this pandemic. His condition was progressively worsening and on third week of his illness he developed right sided tension pneumothorax which is a very unlikely feature of COVID 19. As an emerging global disease every uncommon and new feature of this disease should be noted carefully for proper diagnosis and management of patient and also for awareness of doctors. When there is sudden increase in demand of oxygen of a COVID19 patient do a chest x-ray on urgent to exclude spontenous pneumothorax as an unusual presentation.

\section{References:}

1. "Statement on the second meeting of the International Health Regulations (2005) Emergency Committee regarding the outbreak of novel coronavirus (2019nCoV)". World Health Organization (WHO). 30 January 2020. Archived from the original on 31 January 2020. Retrieved 30 January 2020.

2. "WHO Director-General's opening remarks at the media briefing on COVID-19-11 March 2020". World Health Organization. 11 March 2020. Retrieved 11 March 2020

3 "COVID-19 Dashboard by the Center for Systems Science and Engineering (CSSE) at Johns Hopkins University (JHU)". ArcGIS. Johns Hopkins University. Retrieved 3 August 2020.

4.. A. J. Tyrrell and M. L. Bynoe, "Cultivation of viruses from ahigh proportion of patients with colds," The Lancet, vol. 287,no. 7428, pp. 76-77, 1966.

5. www.cdc.gov>2019symptoms-testing

6, "CT provides best diagnosis for COVID-19". Science Daily. Retrieved 14 March 2020. Institute of Allergy and Infectious Diseases. Retrieved 2 May 2020.

7. Ai T, Yang Z, Hou H, Zhan C, Chen C, Lv W, et al. (February 2020). "Correlation of Chest CT and RT-PCR Testing in Coronavirus Disease 2019 (COVID-19) in China: A Report of 1014 Cases". Radiology. 296 (2): E32E4O. doi:10.1148/radiol.2020200642. PMC 7233399. PMID 32101510 
8. Sanders JM, Monogue ML, Jodlowski TZ, Cutrell JB (April 2020). "Pharmacologic Treatments for Coronavirus Disease 2019 (COVID-19): A Review". JAMA. 323 (18): 1824-1836. doi:10.1001/jama.2020.6019. PMID 32282022. S2CID 215752785

10 "Overview of novel coronavirus (2019-nCoV)—Summary of relevant conditions". The BMJ. Archived from the original on 31 January 2020. Retrieved 1 February 2020.

11. Piechotta, Vanessa; Chai, Khai Li; Valk, Sarah J.; Doree, Carolyn; Monsef, Ina; Wood, Erica M.; Lamikanra, Abigail; Kimber, Catherine; McQuilten, Zoe; So-Osman, Cynthia; Estcourt, Lise J. (10 July 2020). "Convalescent plasma or hyperimmune immunoglobulin for people with COVID19: a living systematic review". The Cochrane Database of Systematic Reviews. 7: CD013600.

12. D. A. J. Tyrrell and M. L. Bynoe, "Cultivation of viruses from a high proportion of patients with colds," The Lancet, vol. 287 , no. 7428 , pp. 76-77, 1966. View at:
"Low-cost dexamethasone reduces death by up to one third in hospitalised patients with severe respiratory complications of COVID-19" (PDF). 16 June 2020. Retrieved 16 June 2020

13. http://www.nih.govcoronavirus. Publisher Site | Google Scholar

14. P. Zhou, X.-L. Yang, X.-G. Wang et al., "A pneumonia outbreak associated with a new coronavirus of probable bat origin," Nature, vol. 579, no. 7798, pp. 270-273, 2020.

15. The Novel Coronavirus Pneumonia Emergency Response Epidemiology Team and China CDC Weekly, "Vital surveillances: the epidemiological characteristics of an outbreak of 2019 novel coronavirus diseases (COVID-19)—China, 2020," China CDC Weekly, vol. 2, no. 8, pp. 113-122, 2020. View at: Publisher Site | Google Scholar

16. (https://www.brighamhealthonamission.org/2020/ 05/14/understanding-unusual-presentations-ofcovid-19/ 